\title{
Evaluation of Host Plant Resistance for Blast and Brown Spot Diseases of Paddy in Hill Zone of Karnataka, India
}

\author{
G.N. Hosagoudar ${ }^{1 *}$, Sheshaiah ${ }^{2}$, Basavaraj S. Kovi ${ }^{2}$ and B.S. Umesh Babu ${ }^{2}$ \\ ${ }^{1}$ AICRIP on Rice, AHRS, Ponnampet, Karanataka, India-571 216 \\ ${ }^{2}$ AHRS, Ponnampet, Karanataka, India-571 216 \\ *Corresponding author
}

\section{Keywords}

Rice, Resistance, Blast, Brown spot and Diseases

Article Info

Accepted:

12 February 2019

Available Online:

10 March 2019

\section{A B S T R A C T}

Rice (OryzasativaL.) is an important cereal crop belonging to the family Poaceae and has two cultivated and 22 wild species. The cultivated species are Oryza sativa and Oryzaglaberrima. Oryza sativa is grown all over the world. Rice is known to suffer from number of diseases caused by fungal, bacterial and viral origins. Identification of resistant genotypes is again an essential continuous process either to recommend for cultivation in endemic area or to use as donors of the resistant genes. In view of these, the present investigation of evaluation of 244 rice varieties/genotypes against leaf and neck blast and brown spot diseases were carried out during Kharif 2014 at AHRS, Ponnampet to identify the sources of resistance under field conditions. 244 entries were tested in Uniform blast screening nursery for leaf blast and transplanted field of screening for neck blast and brown spot diseases resistance of paddy, out of which a IET-23609 found highly resistant to leaf blast disease of paddy and nine entries i.e. IET-23081, 23642, 23616, 23652, 23645, $23612,23637,23300,23299$ showed resistant reactions to the leaf blast and remaining entries were found moderately resistant to highly susceptible reaction to leaf blast. The same entries were also screened for Neck blast disease out of which 38 entries i.e., IET23088, 23081, 23642, 23616, 23652, Swarna, 23627, 23645, 23612, 23654, 23275, 24132, 24151, 23224, 23758, 24116, 23739, 22654, 23052, 23055, 23053, 23601, 23599, 23594, 23591, 23004, 22986, 22989, Dinesh, 22302, 22994, 23422, 22878, 24074, 24167, Samba Mahsuri (RP-1), Improved Samba Mahsuri (RP-2) and 23883 were found highly resistant, remaining entries were found resistant to highly susceptible reaction to Neck blast. Similarly, the same entries were also screened for brown spot disease out of which none of the entries found highly resistance and 3 entries i.e., IET-23403, 22876 and 23392 were found resistant reaction to brown spot disease. The remaining entries were found moderately resistant to highly susceptible reaction to brown spot disease.

\section{Introduction}

Rice (OryzasativaL.) is an important cereal crop belonging to the family Poaceae. Rice is the most important staple food crop and grown in India providing of 43 per cent of calorie requirement for 70 per cent of the Indian population. India is the largest rice growing country accounting for about one third of the world acreage under the crop. In India, annual 
rice production is $103.6 \mathrm{mt}$ during 2016 (Anon, 2016). The major rice growing states of India are West Bengal, Bihar, Madhya Pradesh, Orissa, Andhra Pradesh, Karnataka and Uttar Pradesh, which accounts 69.80 per cent of the total area.

Rice suffers from many biotic and abiotic factors which result in the lower productivity. Among the biotic factor it suffer from fungal diseases viz., blast (Pyricularia oryzae), brown leaf spot (Bipolaris oryzae / Helminthosporium oryzae), stem rot (Sclerotium oryzae), sheath blight (Rhizoctonia solani), sheath rot (Sarocladium oryzae), bacterial disease such as bacterial blight (Xanthomonas oryzaepv. oryzae) and viral disease such as (rice tungro virus) nematode disease such as rice root knot (Meloidogyne graminicola) are important. Among fungal diseases blast and brown spot disease is of economic importance. In India, the disease was first recorded in Thanjavore delta of south India in 1918 by Mc Rae (1922). However, it attracted the attention only when a devastating epidemic occurred in 1919 (Padmanabhan, 1965). Among the fungal disease, brown leaf spot of rice incited by Helminthosporoum oryzae is a major disease occurring in almost all the rice growing areas of the world causing 5 per cent yield loss across all lowland rice production situations in south and Southeast Asia (Savary, 2000).

The pathogen attacks all the aerial parts of plants at any stage of crop growth right from germination to harvest. The disease occurs as seedling blight, leaf blast, node blast, neck or panicle blast and grain spot. Seed and soil borne infection during germination and thereafter on tender seedling cause, seedling blight leading to death of seedling. Leaf blast is characterized by production of large spindle shaped lesions with ashy grey centres with brown margins drastically reduce crop growth and tillering. The infected node or neck tissues became soft and rotted. The node or neck blast is considered as the most destructive phase of the disease attacking prior or after flowering and grain formation, causing drastic reduction in grain quality and quantity of produce. The infection on grain produced dark brownish black spot.

Blast disease commonly occurs in few paddy growing areas like Mandya, Kodagu, Shivamogga, Uttara Kannada, Dakshina Kannada, Mysore and Chickmagaluru districts (Anon., 1981). The occurrence of disease in moderate to severe form in hilly areas of Karnataka is mainly due to indiscriminate use of nitrogenous fertilizers, heavy rainfall, mono-cropping and repeated cultivation of the crop. This leads to severe losses in grain yield of the crop. Moreover, repeated cultivation of paddy would not only make the higher inoculum build up but also aggravate the disease problem.

Cultivation of resistant genotypes is the effective and cheaper method to combat the disease as compared to the chemical control. Hence, several genotypes should be screened to identify the resistant genotypes. In view of these, the present investigation of evaluation of 244 rice varieties/genotypes against blast and brown spot diseases were carried out during Kharif 2014 at AHRS, Ponnampet to identify the sources of resistance under field conditions.

\section{Materials and Methods}

Screening of rice genotypes against leaf blast disease in Uniform Blast Nursery (UBN) pattern

The experiment was conducted during Kharif 2014 at AHRS, Ponnampet. Screening of rice against $P$. oryzae was carried out to know the source of resistance against leaf blast disease 
under natural epiphytotic condition. 244 rice germplasm lines were evaluated against leaf blast disease. These germplasm lines were sown on 07-07-2014 in UBN (Uniform Blast Nursery) pattern. It is an identification of naturally existing blast strains in the hotspot locality. In this technique, nursery beds of 0.5 $\mathrm{m}$ width and length of $50 \mathrm{~m}$ were raised using spade, all around the bed the most susceptible checks were sown to serve as a source of inoculums for spreading the disease. The susceptible and resistant checks were sown initially and each test entry were sown at 10 $\mathrm{cm}$ apart in one line and after every 20 test entries again susceptible and resistant checks were sown. Disease reactions were recorded by using $0-9$ scale given below.

Scoring for leaf blast was done at nursery stage by using following scale given by IRRI (1996)

\begin{tabular}{|c|c|c|}
\hline Rating scale & Disease severity & Host response \\
\hline 0 & No lesion observed & Highly Resistant \\
\hline 1 & Small brown specks of pin point size & Resistant \\
\hline 2 & $\begin{array}{l}\text { Small roundish to slightly elongated, necrotic grey } \\
\text { spots, about } 1-2 \mathrm{~mm} \text { in diameter, with a distinct brown } \\
\text { margin. Lesions are mostly found on the lower leaves }\end{array}$ & $\begin{array}{l}\text { Moderately } \\
\text { Resistant }\end{array}$ \\
\hline 3 & $\begin{array}{l}\text { Lesion type same as in } 2 \text {, but significant number of } \\
\text { lesions on the upper leaves }\end{array}$ & $\begin{array}{l}\text { Moderately } \\
\text { Resistant }\end{array}$ \\
\hline 4 & $\begin{array}{l}\text { Typical susceptible blast lesions, } 3 \mathrm{~mm} \text { or longer } \\
\text { infecting less than } 4 \% \text { of leaf area }\end{array}$ & $\begin{array}{l}\text { Moderately } \\
\text { Susceptible }\end{array}$ \\
\hline 5 & $\begin{array}{l}\text { Typical susceptible blast lesions of } 3 \mathrm{~mm} \text { or longer } \\
\text { infecting } 4-10 \% \text { of the leaf area }\end{array}$ & $\begin{array}{l}\text { Moderately } \\
\text { Susceptible }\end{array}$ \\
\hline 6 & $\begin{array}{l}\text { Typical susceptible blast lesions of } 3 \mathrm{~mm} \text { or longer } \\
\text { infecting } 11-25 \% \text { of the leaf area }\end{array}$ & $\begin{array}{l}\text { Moderately } \\
\text { Susceptible }\end{array}$ \\
\hline 7 & $\begin{array}{l}\text { Typical susceptible blast lesions of } 3 \mathrm{~mm} \text { or longer } \\
\text { infecting } 26-50 \% \text { of the leaf area }\end{array}$ & Susceptible \\
\hline 8 & $\begin{array}{l}\text { Typical susceptible blast lesions of } 3 \mathrm{~mm} \text { or longer } \\
\text { infecting } 51-75 \% \text { of the leaf area many leaves are dead }\end{array}$ & $\begin{array}{l}\text { Highly } \\
\text { Susceptible }\end{array}$ \\
\hline 9 & $\begin{array}{l}\text { Typical susceptible blast lesions of } 3 \mathrm{~mm} \text { or longer } \\
\text { infecting more than } 75 \% \text { leaf area affected }\end{array}$ & $\begin{array}{l}\text { Highly } \\
\text { Susceptible }\end{array}$ \\
\hline
\end{tabular}

Screening of rice genotypes against neck blast and brown spot diseases in field nursery pattern

Another experiment was conducted during Kharif2014 at AHRS, Ponnampet. Screening of rice against $P$. oryzae and Helminthosporium oryzae was carried out to know the source of resistance against neck blast and brown spot diseases under natural epiphytotic condition. 244 rice germplasm lines were evaluated against neck blast and brown spot diseases. These germplasm lines were sown on 01-07-2014 in field nursery pattern. Intan and IR-64 serve as susceptible and resistant check respectively. One line of 25 days old seedlings were uprooted from the nursery bed and planted in the main field over a length of 1.5 meters in two lines with a spacing of $15 \times 15 \mathrm{~cm}$. Fertilizers were applied at the rate of 75:75:90 Kg/ha i.e. basal application at the rate of $37.5: 75: 45 \mathrm{Kg} / \mathrm{ha}$ and top dressing of $37.5: 0: 45 \mathrm{Kg} /$ ha respectively. The screening against neck blast and brown spot resistance and disease reactions were recorded using 0-9 scale given below. 
Scoring for neck blast was done at harvesting stage by using following scale given by IRRI (1996)

\begin{tabular}{|c|c|c|}
\hline Rating scale & Disease Reaction & Description \\
\hline 0 & HR & No incidence \\
\hline 1 & R & Less than $5 \%$ infected panicles \\
\hline 3 & MR & $5-10 \%$ infected panicles \\
\hline 5 & MS & $11-25 \%$ infected panicles \\
\hline 7 & S & $26-50 \%$ infected panicles \\
\hline 9 & HS & More than $50 \%$ infected panicles \\
\hline
\end{tabular}

Scoring for brown spot by using following scale given by IRRI (1996)

\begin{tabular}{|c|c|c|}
\hline Rating scale & $\begin{array}{c}\text { Disease severity in per } \\
\text { cent leaf area coverage }\end{array}$ & Host response \\
\hline 0 & No incidence & Highly Resistant \\
\hline 1 & Less than $1 \%$ & Resistant \\
\hline 2 & $1-3 \%$ & Moderately Resistant \\
\hline 3 & $4-5 \%$ & Moderately Resistant \\
\hline 4 & $6-10 \%$ & Moderately Susceptible \\
\hline 5 & $11-15 \%$ & Moderately Susceptible \\
\hline 6 & $16-25 \%$ & Moderately Susceptible \\
\hline 7 & $26-50 \%$ & Susceptible \\
\hline 8 & $51-75 \%$ & Highly Susceptible \\
\hline 9 & $76-100 \%$ & Highly Susceptible \\
\hline
\end{tabular}

\section{Results and Discussion}

Screening of rice genotypes against blastand brown spot diseases in Nurseries

In order to identify the resistant sources, 244 rice genotypes were screened by using 0-9 scale against leaf and neck blast caused by $P$. oryzae under natural epiphytotic condition at AHRS, Ponnampet. The genotypes were grouped into six classes based on degree of reaction and the number of genotypes falling in particular group and results are presented in Table 1 and Plate 1 respectively.

Among 244 genotypes screened against leaf blast, out of which a IET-23609 found highly resistant to leaf blast disease of paddy, nine entries i.e.,IET-23081, 23642, 23616, 23652,
23645, 23612, 23637, 23300 and 23299showed resistant reactions to the leaf blast, thirty one entries i.e.,IET-23088, swarna, 23627, 23654, 23275, 23324, 23682, 23666, 23695, P A 6129, IET-24133, 23714, 23680, 23230, 23758, 23223, 24111, 1 R 64, 23216, 23831, 23834, 23345, Sahbhagidhan, 23341, 24075, 23356, 23977, 23983, 23949, 23979 and 23993 showed moderately resistant reactions to the leaf blast. One hundred forty eight entries i.e.,IET-23272, 23720, 23687 , 24132, 24143, 24151, 24138, 23224, 24098, 24103, 24116, 23770, 24104, 24120, 24097, 24100, 23743, US 312, 22654, Kalanamak, 23203, 23830, 23824, IR 64, 23333, 22704, 23448, 23409, 23420, 22876, 23392, 2339, 24074, 23984, 23988, 23951, 24081, 24082, 2503, 2505, 2506,24576, 24577, PusaSugandh 5 (RP), Check Variety (HR-12, T (N)1), IET- 
24142, 24126, 24147, 24146, NDR 359, 23268, 23739, 23777, 23735, 24122, 24113, 23017, 23596, 23603, Jalmagna, 22302, 23193, Chittimuthyalu, 23832, Samba Mahsuri, 23329, 23334, 23337, 24003, 24001, C R Dhan 201, 23459, 23466, 23471,23422, 22878, Sahbhagidhan, 23435, P A 6129, 23947, 23354, 23976, 23996, 24565, 24566, 24567, 24572, Pusa Basmati 6 (RP),24575, 24578, Check Variety (IR-64 \& IR-50), 24166, C 101 LAC (DP 2), 24164, 23878, IET-22920, 23678, 23226, 22913, NDR 359, 23227, 24114, 24117, Sabita, 23053, 23004,23010, Dinesh, 23009, 22648, 23829, Kalanamak, 22747, 23355, 24049, 24051, PA6129, 23997, 24010, CR Dhan 201, 24008, 23450, 23449, 23455, 22716, 23427, 23412, 23429, 23417, 23425, 23982, 23992, 23957, 23806, 23362, 24569, 24570, 23805, Check Variety (CO-39, CH-45), 24102, SanbaMahsuri (RP1), Tetep (DP 1), 24232, 23864 and 23883 showed moderately susceptible reactions to the leaf blast. Twenty six entries i.e., IET-23235, 23052, 23601, 23599, 23594, 22986, 22989, 22994, 22649, 24056, 24035, 24038, 24016, 24037, 23467, 23445, 22770, 23421, 23959, 24571, 23804, 24573, Check Variety (Benibhog), 24099, 24167and 23879 showed susceptible reactions to the leaf blast. Twenty five entries i.e., IET24093, 23055, 23591, 23194, Badshabhog, 23189, 24006, 24025, 24028, 24007, 24036, 23463, 23430, 23413, 23411, 24568, 23367, 24574, Check Varieties (Nidhi, Vikramarya, Ajaya, Swarnadhan), Improved Samba Mahsuri (RP 2), Taroari Basmati and Check Variety (RP-Bio-26) showed highly susceptible reaction to leaf blast respectively (Table 1).

Among 244 genotypes screened against neck blast, out of which thirty eight entries i.e., IET23088, 23081, 23642, 23616, 23652, Swarna, 23627, 23645, 23612, 23654, 23275, 24132, 24151, 23224, 23758, 24116, 23739, 22654, 23052, 23055, 23053, 23601, 23599, 23594,
23591, 23004, 22986, 22989, Dinesh, 22302, 22994, 23422, 22878, 24074, 24167, Samba Mahsuri (RP-1), Improved Samba Mahsuri (RP-2) and 23883found highly resistant to neck blast disease of paddy, Fifty eight entries i.e.,IET-23637, 23299, 23324, 23682, 23666, 23714, 24142, 22920, 24126, 23687, 24143, 24147, 24146, 23226, NDR-359, 23227 , 23268, 23230, 24120, 24122, 24113, US-312, 23017, 23596, 23603, Jalmagna, 23010, Badshabhog, 23189, 22649, Kalanamak, 23832, Samba Mahsuri, 23834, 23337, 23355, PA-6129, 24006, 24028, 24007, 23445, 22716, PA-6129, 23982, 22876, 23976, PA6129, 24082, 24568, 23804, Check Variety (Rasi, RP-Bio-226, CO-39, Benibhog), C101LAC (DP2), 24232, 23864 and 23879 showed resistant reactions to the neck blast,Fifty eight entries i.e., IET-23609, 23647, 23272, 23680, 23720, NDR-359, 23678, 23235, 22913, 23223, IR-64, 23770, 24100, 24114, 24093, 23743, 23735, 24117, Purnendu, 23009, 22648, 23194, 23203, IR64, Chittimuthyalu, 23831, 23329, 23345, 24003, 24001, 22704, 23448, 23471, 23409, 23429, 23413, 23947, 23988, 24075, 23356, 22977, 23983, 23951, 23354, 23993, 24567, 24577, Check Variety (Nidhi, Vikramarya, Ajay, Swarnadhan, CH-45, IR-50), 24102, Tetep (DP-1), 24166, 24164 and 23878 showed moderately resistant reactions to the neck blast.Seventy one entries i.e., IR-64, IET-23300, 24133， 23695，24138， 24098, 24103, 24111, 23777, 24104, 24097, 23216, Sabita, 23193, 23830, 23834, 23829, 22747, 23333, Sahbhagidhan, 23334, 24049, 24051, 24056, 23997, 24010, 24038, 24025, 24016, 24036, 24037, 23463, 23467, 23450, 23449, 23466, 23455, 23427, Sahbhagidhan, 23420, 23435, 23425, 23411, 23959, 23392, 23984, 23341, 23992, 23996, 24081, 23949, 23957, 23806, 23362, 24562, 24563, 24564, 24565, 24566, 24570, 24571, Taroari Basmati, 23367, 23805, 24574, Pusa Basmati-6 (RP), 24576, 24578, Check Variety (IR-64, T(N)-1) and 24099 showed moderately susceptible 
reactions to the neck blast. Eighteen entries i.e., Kalanamak, IET-24035, CR Dhan-201, 24008, CR Dhan-201, 23459, 22770, 23355, 23417, 23430, 23421, 23339, 23979, 24569, 24572, 24573, 24575 and Pusa Sugandh-5 (RP) showed highly susceptible reaction to neck blast respectively (Table 2 and Plate 2). Intan served as susceptible check showed highly susceptible and susceptible reaction to leaf and neck blast respectively. IR-64 served as resistant check showed moderately resistant reactions against leaf and neck blast respectively. To identify the resistant sources against the any disease in order to breed resistant varieties is of primary importance. Wide response of rice genotypes against $P$. oryzae has been earlier observed by various workers (Nagaraju et al., 1991, Saifulla and Manjunath, 1995 and Ghazanfar et al., 2009). Saha (2004) reported that HR-12 showed susceptibility to $P$. oryzae as observed in the present study. Ravi et al., (1989) reported that Jaya variety showed high degree of resistant to the blast of rice. The results obtained in the present study are supported by Naiket al., (2016) who reported that Intan and HR-12 showed highly susceptible reaction to blast of rice, while Rasi and IR-64 recorded moderately susceptible reaction to the blast of rice. Barnwal et al., (2012) reported and used C0-39 as susceptible variety to blast of rice in experiment to evaluate fungicides.

Among 244 genotypes screened against brown spot, out of which none of the entries found highly resistant to brown spot disease of paddy, three entries i.e.,IET-23403, 22876 and 23392 showed resistant reactions to the leaf blast, Seventy entries i.e.,IET-22654, 23599, Kalanamak, 23333，23355，24025，23459, 23466, 23427, 22770, Sahbhagidhan, 23412, 23425, 23413, 23411, 23947, 23983, 23949, 23979, 24572, 23804, 24576, Check Variety (HR-12, Rasi, IR-64, T(N)-1, CH-45), Samba Mahsuri (RP-1), Tetep (DP-1), 24164, 24232, IET-23739，23052，23055，23053， 23601,
23594, 22986, 23010, 23194, Samba Mahsuri, 23834, 44049, 24051, 24008, 24028, 24016, 22704, 23449, 23420, 23435, 23421, 23959, 23339, 23984, 23341, 24562, 24568, 24569, 24570, 24571, Taroari Basmati,, 23367, 23805, 24575, Check Variety (Co-39, Benibhog), 24167, C 101LAC(DP-2) and Improved Samba Mahsuri (RP-2) showed moderately resistant reactions to the brown spot disease. One hundred thirty seven entries i.e., IET-23642, 23645, 23637, 23714, 23272, 24147, 24146, 23224, 23227, 24098, 23223, 23216, 24100, 23017, 23004, 22989, Dinesh, 23203, 23830, 23831, 23829, 22747, 23345, 24038, CR Dhan-201, 24007, 24036, 23467, 23450, 23471, 23455, 23992, 23976, 23996, 24564, 24565, 24566, 24567, 24574, Check Variety (RP-Bio-226), IET-23652, 23647, 23300, 24133, 23666, 22920, 24132, 24143, 23230, 24111, 24104, 24097, 24114, 24117, Purnendu, 22302, 22994, 22648, 23824, IR64, 23329, 23997, 24037, 23463, 23445, $22704,23448,23422,23409,23417$, PA6129, 23988, 23356, 23977, 23951, 23354, 23993, 23362, Pusa Basmati-6 (RP), Pusa Sugandh-5 (RP), Check Variety (Vikramarya, Ajaya, IR-50), 24099, 24102, 23864, 23878, 23879, IET-23616, 23609, 23612, 23654, IR64, 23275, 23682, 24126, 23720, 23687, 24151, 23695, 24138, 23678, 23268, 23758, 24116, 23770, 24093, 23743, 23735, 24122, US-312, 23596, 23591, 23189, 22649, 23193, 23832, 23334, 23337, 24056, 24035, 24006, 24010, 24003, 24001, 22716, 23409, 23429, 23982, 24075, 24081, 23957, 23806, 24573, 24578, Check Variety (Nidhi) and 24166 showed moderately susceptible reactions to the brown spot disease. Twenty seven entries i.e., IET-23088, 23081, Swarna, 23299, 23324, 24142, 23680, 23226, 22913, 23227, 23777, 24120, 24113, Sabita, 23603, Jalmagna, Badshabhog, Kalanamak, Chittimuthyalu, Sahbhagidhan, PA-6129, 24074, PA-6129, 24082, 24563, 24577 and 23883 showed susceptible reactions to the brown spot disease. Six entries i.e., IET- 
23627, NDR-359, 23235, NDR-359, 24103 and 23009showd highly susceptible reaction to the brown spot disease respectively (Table
2 and Plate 2). Wide response of rice genotypes against Helminthosporoum oryzae has been earlier observed by various workers.

Table.1 Grouping of paddy genotypes based on their reaction to Leaf blast under Nursery (NSN1) conditions

\begin{tabular}{|c|c|}
\hline $\begin{array}{l}\text { Reaction } \\
\text { (SES 0-9 } \\
\text { Scale) }\end{array}$ & Entry No. (IET) \\
\hline $\mathbf{0}(\mathbf{H R})$ & (1) IET-23609 \\
\hline $1(\mathbf{R})$ & (9) IET-23081, 23642, 23616, 23652, 23645, 23612, 23637, 23300, 23299 \\
\hline 2(MR) & $\begin{array}{l}\text { (10) IET-23088, swarna, 23627, 23654, 23275, 23324, 23682, 23666, 23695, P A } \\
6129\end{array}$ \\
\hline 3 (MR) & $\begin{array}{l}\text { (21) IET-24133, 23714, 23680, 23230, 23758, 23223, 24111, } 1 \text { R 64, 23216, } \\
23831,23834,23345 \text {, Sahbhagidhan, 23341, 24075, 23356, 23977, 23983, 23949, } \\
23979,23993\end{array}$ \\
\hline $4(\mathrm{MS})$ & $\begin{array}{l}\text { (46) IET-23272, 23720, 23687, 24132, 24143, 24151, 24138, 23224, 24098, } \\
\text { 24103, 24116, 23770, 24104, 24120, 24097, 24100, 23743, US 312, 22654, } \\
\text { Kalanamak, 23203, 23830, 23824, IR 64, 23333, 22704, 23448, 23409, 23420, } \\
\text { 22876, 23392, 2339, 24074, 23984, 23988, 23951, 24081, 24082, 2503, 2505, } \\
\text { 2506,24576, 24577, PusaSugandh 5 (RP), Check Variety (HR-12, T (N)1) }\end{array}$ \\
\hline 5 (MS) & $\begin{array}{l}\text { (51) IET-24142, 24126, 24147, 24146, NDR 359, 23268, 23739, 23777, 23735, } \\
\text { 24122, 24113, 23017, 23596, 23603, Jalmagna, 22302, 23193, Chittimuthyalu, } \\
\text { 23832, Samba Mahsuri, 23329, 23334, 23337, 24003, 24001, C R Dhan 201, } \\
\text { 23459, 23466, 23471,23422, 22878, Sahbhagidhan,23435, P A 6129, 23947, } \\
\text { 23354, 23976, 23996, 24565, 24566, 24567, 24572, Pusa Basmati } 6 \text { (RP),24575, } \\
\text { 24578, Check Variety (IR-64\&IR-50), 24166, C 101 LAC (DP 2), 24164, 23878, }\end{array}$ \\
\hline 6 (MS) & $\begin{array}{l}\text { (51) IET-22920, 23678, 23226, 22913, NDR 359, 23227, 24114, 24117, Sabita, } \\
\text { 23053,23004,23010,Dinesh,23009,22648,23829,Kalanamak,22747,23355,24049,2 } \\
\text { 4051,PA6129,23997,24010,CRDhan201,24008,23450,23449,23455,22716,23427, } \\
\text { 23412,23429,23417,23425,23982,23992,23957,23806,23362,24569,24570,23805, } \\
\text { Check Variety (CO-39, CH-45), 24102,Sanba Mahsuri (RP1),Tetep (DP } \\
\text { 1),24232,23864,23883, }\end{array}$ \\
\hline $7(S)$ & $\begin{array}{l}\text { (26) IET- } \\
\text { 23235,23052,23601,23599,23594,22986,22989,22994,22649,24056,24035,24038, } \\
\text { 24016,24037,23467,23445,22770,23421,23959,24571,23804,24573,Check Variety } \\
\text { (Benibhog), 24099,24167,23879, }\end{array}$ \\
\hline 8 (HS) & $\begin{array}{l}\text { (23)IET-24093, 23055, 23591, 23194, Badshabhog, 23189, 24006, 24025, 24028, } \\
\text { 24007, 24036, 23463, 23430, 23413, 23411, 24568, 23367, 24574, Check Variety } \\
\text { (Nidhi, Vikramarya, Ajaya, Swarnadhan), Improved Samba Mahsuri (RP 2), }\end{array}$ \\
\hline 9 (HS) & (2) Taroari Basmati, Check Variety (RP-Bio-26), \\
\hline
\end{tabular}


Table. 2 Grouping of paddy genotypes based on their reaction to neck blast \& brown spot under transplanted field conditions

\begin{tabular}{|c|c|c|}
\hline \multirow{2}{*}{$\begin{array}{l}\text { Reaction } \\
\text { (SES 0-9 } \\
\text { Scale) }\end{array}$} & \multicolumn{2}{|c|}{ Entry No. (IET) } \\
\hline & Neck blast & Brown spot \\
\hline O (HR) & $\begin{array}{l}\text { (38) IET-23088, 23081, 23642, 23616, } \\
\text { 23652, Swarna, 23627, 23645, 23612, 23654, } \\
\text { 23275, 24132, 24151, 23224, 23758, 24116, } \\
\text { 23739, 22654, 23052, 23055, 23053, 23601, } \\
\text { 23599, 23594, 23591, 23004, 22986, 22989, } \\
\text { Dinesh, 22302, 22994, 23422, 22878, 24074, } \\
\text { 24167, Samba Mahsuri (RP-1), Improved } \\
\text { Samba Mahsuri (RP-2) and 23883 }\end{array}$ & - \\
\hline $1(\mathbf{R})$ & $\begin{array}{l}\text { (58) IET-23637, 23299, 23324, 23682, } \\
\text { 23666, 23714, 24142, 22920, 24126, 23687, } \\
\text { 24143, 24147, 24146, 23226, NDR-359, } \\
23227,23268,23230,24120,24122,24113, \\
\text { US-312, 23017, 23596, 23603, Jalmagna, } \\
\text { 23010, Badshabhog, 23189, 22649, } \\
\text { Kalanamak, 23832, Samba Mahsuri, 23834, } \\
\text { 23337, 23355, PA-6129, 24006, 24028, } \\
24007,23445,22716, \text { PA-6129, 23982, } \\
\text { 22876, 23976, PA-6129, 24082, 24568, } \\
\text { 23804, Check Variety (Rasi, RP-Bio-226, } \\
\text { CO-39, Benibhog) C-101LAC (DP2), } \\
\text { 24232, 23864 and 23879 }\end{array}$ & (3) IET-23403, 22876 and 23392 \\
\hline 2 (MR) & & $\begin{array}{l}\text { (31) IET-22654, 23599, Kalanamak, 23333, 23355, } \\
\text { 24025, 23459, 23466, 23427, 22770, Sahbhagidhan, } \\
\text { 23412, 23425, 23413, 23411, 23947, 23983, 23949, } \\
\text { 23979, 24572, 23804, 24576, Check Variety (HR- } \\
\text { 12, Rasi, IR-64, T(N)-1, CH-45), Samba Mahsuri } \\
\text { (RP-1), Tetep (DP-1), 24164 and 24232 }\end{array}$ \\
\hline 3 (MR) & $\begin{array}{l}\text { (58) IET-23609, 23647, 23272, 23680, } \\
\text { 23720, NDR-359, 23678, 23235, 22913, } \\
\text { 23223, IR-64, 23770, 24100, 24114, 24093, } \\
\text { 23743, 23735, 24117, Purnendu, 23009, } \\
\text { 22648, 23194, 23203, IR-64, Chittimuthyalu, } \\
\text { 23831, 23329, 23345, 24003, 24001, 22704, } \\
\text { 23448, 23471, 23409, 23429, 23413, 23947, } \\
\text { 23988, 24075, 23356, 22977, 23983, 23951, } \\
\text { 23354, 23993, 24567, 24577, Check Variety } \\
\text { (Nidhi, Vikramarya, Ajay, Swarnadhan, CH- } \\
\text { 45, IR-50), 24102, Tetep (DP-1), 24166, } \\
\text { 24164 and 23878, }\end{array}$ & $\begin{array}{l}\text { (39) IET-23739, 23052, 23055, 23053, 23601, } \\
23594,22986,23010,23194, \text { Samba Mahsuri, } \\
\text { 23834, 44049, 24051, 24008, 24028, 24016, 22704, } \\
\text { 23449, 23420, 23435, 23421, 23959, 23339, 23984, } \\
\text { 23341, 24562, 24568, 24569, 24570, 24571, Taroari } \\
\text { Basmati,, 23367, 23805, 24575, Check Variety (Co- } \\
\text { 39, Benibhog), 24167, C 101LAC(DP-2) and } \\
\text { Improved Samba Mahsuri (RP-2), }\end{array}$ \\
\hline 4 (MS) & & $\begin{array}{l}\text { (40) IET-23642, 23645, 23637, 23714, 23272, } \\
24147,24146,23224,23227,24098,23223,23216, \\
24100,23017,23004,22989, \text { Dinesh, 23203, } \\
23830,23831,23829,22747,23345,24038, \text { CR } \\
\text { Dhan-201, 24007, 24036, 23467, 23450, 23471, } \\
\text { 23455, 23992, 23976, 23996, 24564, 24565, 24566, } \\
24567,24574 \text { and Check Variety (RP-Bio-226) }\end{array}$ \\
\hline 5 (MS) & $\begin{array}{l}\text { (71) IR-64, IET-23300, 24133, 23695, } \\
24138,24098,24103,24111,23777,24104, \\
24097,23216, \text { Sabita, 23193, 23830, 23834, }\end{array}$ & $\begin{array}{l}\text { (48) IET-23652, 23647, 23300, 24133, 23666, } \\
22920,24132,24143,23230,24111,24104,24097, \\
24114,24117, \text { Purnendu, 22302, 22994, 22648, }\end{array}$ \\
\hline
\end{tabular}




\begin{tabular}{|c|c|c|}
\hline & 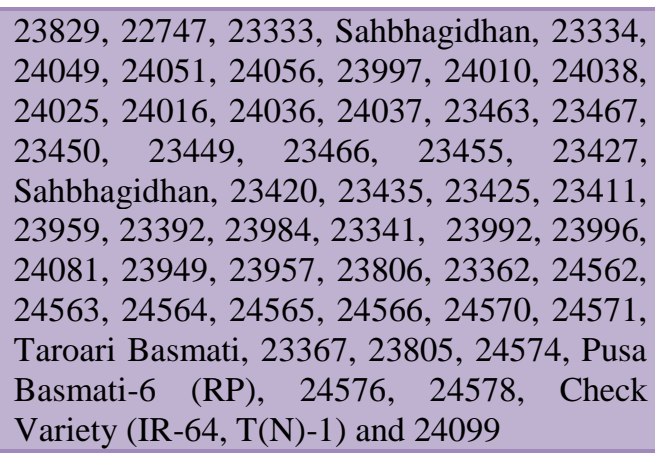 & $\begin{array}{l}\text { 23824, IR-64, 23329, 23997, 24037, 23463, 23445, } \\
22704,23448,23422,23409,23417, \text { PA-6129, } \\
\text { 23988, 23356, 23977, 23951, 23354, 23993, 23362, } \\
\text { Pusa Basmati-6 (RP), Pusa Sugandh-5 (RP), Check } \\
\text { Variety (Vikramarya, Ajaya, IR-50), 24099, 24102, } \\
\text { 23864, } 23878 \text { and 23879 }\end{array}$ \\
\hline 6 (MS) & & $\begin{array}{l}\text { (49) IET-23616, 23609, 23612, 23654, IR-64, } \\
23275,23682,24126,23720,23687,24151,23695, \\
24138,23678,23268,23758,24116,23770,24093, \\
23743,23735,24122, \text { US-312, 23596, 23591, } \\
23189,22649,23193,23832,23334,23337,24056, \\
24035,24006,24010,24003,24001,22716,23409, \\
23429,23982,24075,24081,23957,23806,24573, \\
\text { 24578, Check Variety (Nidhi) and 24166 }\end{array}$ \\
\hline $7(S)$ & - & $\begin{array}{l}\text { (27) IET-23088, 23081, Swarna, 23299, 23324, } \\
\text { 24142, 23680, 23226, 22913, 23227, 23777, 24120, } \\
\text { 24113, Sabita, 23603, Jalmagna, Badshabhog, } \\
\text { Kalanamak, Chittimuthyalu, Sahbhagidhan, PA- } \\
6129,24074, \text { PA-6129, 24082, 24563, } 24577 \text { and } \\
23883\end{array}$ \\
\hline 8 (HS) & & $\begin{array}{l}\text { (6) IET-23627, NDR-359, 23235, NDR-359, } 24103 \\
\text { and } 23009\end{array}$ \\
\hline 9 (HS) & $\begin{array}{l}\text { (18) Kalanamak, IET-24035, CR Dhan-201, } \\
\text { 24008, CR Dhan-201, 23459, 22770, 23355, } \\
\text { 23417, 23430, 23421, 23339, 23979, 24569, } \\
\text { 24572, 24573, 24575 and Pusa Sugandh-5 } \\
\text { (RP) }\end{array}$ & - \\
\hline
\end{tabular}

Plate.1 screening of rice genotypes against leaf blast disease in Uniform Blast Nursery (UBN) pattern

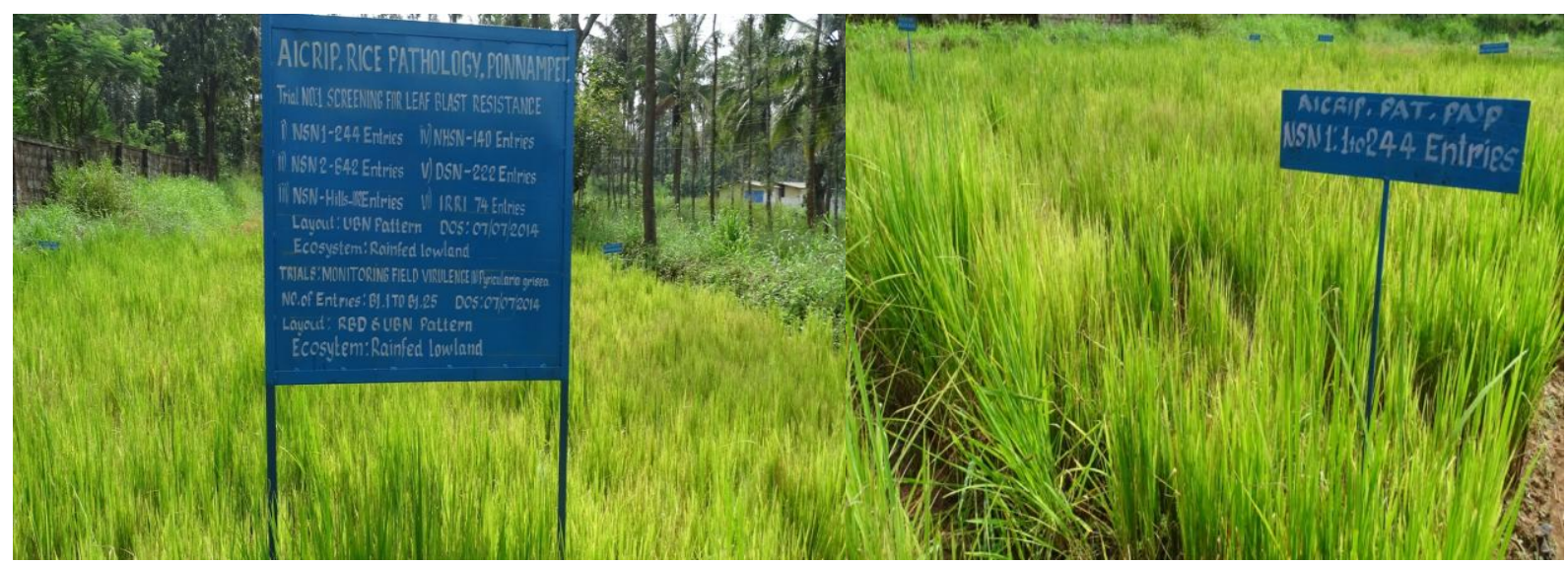


Plate.2 screening of rice genotypes against neck blast and brown spot diseases in field nursery

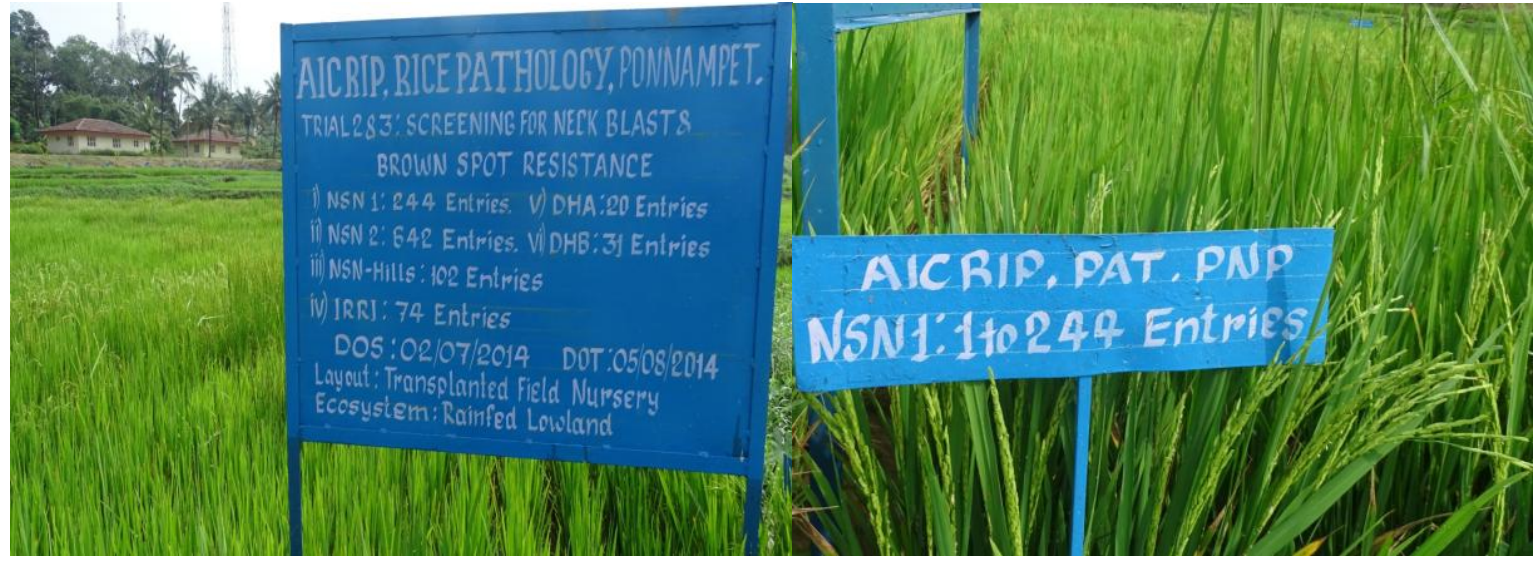

Arshad et al., (2008) were evaluated seventy genotypes/entries/varieties, among that only one Basmati entry, PK-3699-43 of PARC was found resistant, while all other remaining varieties/entries were found moderately resistant to highly susceptible.

Mother et al., (2013) screened five rice cultivars, one hybrid (WR96), three modern (BR16, BR26, and BRRI Dhan27) and one local (Pari) were screened for their reaction to brown leaf spot disease caused by Cochliobolus miyabeanus and their performance on yield-related characters. The severity of brown leaf spot varied with growth stages of rice plant as well as different cultivars tested under field condition. Low disease severity was observed at maximum tillering stage compared to moderate to high at dough stage, with hybrid cultivar WR96 showing highest disease, while local cultivar Pari had the lowest.

\section{References}

Anonymous, 1981, Package of practices for high yields. Univ. Agric. Sci. Bangalore.

Anonymous, 2016, Statistical database. http://www.fao.org

Barnwal, M. K., Vaibhav, K. S., Sharma, R. B. and Singh, B. N., 2012, Field evaluation of rice genotypes for resistance and new fungicides for control of blast (Pyricularia oryzae). Indian Phytopathol., 65(1): 56-59.

Ghazanfar, M. U., Wakil, W., Sahi, S. T. and Yasin, S., 2009, Influence of various fungicides on the management of rice blast disease. Mycopath, 7(1): 29-34.

International Rice Research Institute (IRRI), 1996, Standard evaluation system for rice. 4th.ed. IRRI, Manila, Phillipine.

Mcrae, W., 1922, Report of the Imperial Mycologist Scientific Report, Pusa Agricultural Research Institute, p. 4450.

Nagaraju, P., Vasantakumar, H. C., Devaiah, B. M., Seshadri, V. S. and Naidu, B. S., 1991, Evaluation of rice genotypes for blast and sheath rot resistance in hilly regions of Karnataka, Mysore J. Agric. Sci., 25: 139-141.

Naik, G. R., Dinesh, K. M., Naik, G. B. and Naik, B. T., 2016, Field evaluation of high yielding and local paddy cultivars of farmers seeds source in southern transitional zone (zone-7) of Karnataka against blast disease. I.J.A.B.R., 6(1): 21-24.

Padmanabhan, S. Y., 1965, Breeding for blast resistance in India. In 'The rice blast disease', Ed. Johns Hopkins Press, Baltimore and Maryland, USA, pp. 203- 
221.

Ravi, K., Mishra, R. P. and Diwakar, M. C., 1989, Field reaction of some varieties to blast in Karnataka., Pl. Prot. Bull., Faridabad, 4: 13-14.

Saha, S., 2004, Behavior of rice varieties against leaf blast disease in rainfed upland conditions of West Bengal. $J$. Mycopathol. Res., 42(2): 205-206.

Saifulla, M. and Manjunath, A., 1995, Assessment of rice genotypes to blast disease. Agric. Sci. Dig., 15: 151-152.

Arshad, H. M. I., Khan, J. A. and Jamil, F.F., 2008, Screening of rice germplasm against blast and brown spot disease. Pak. J.Phytopathol., 20(1): 52-57.

Savary, R. A., 1959, Nomenclature of Drechslera and Bipolaris, grass parasites segregated from Helminthosporium candisian J.Bot., 37:879-87.

\section{How to cite this article:}

Hosagoudar, G.N., Sheshaiah, Basavaraj S. Kovi and Umesh Babu, B.S. 2019. Evaluation of Host Plant Resistance for Blast and Brown Spot Diseases of Paddy in Hill Zone of Karnataka, India. Int.J.Curr.Microbiol.App.Sci. 8(03): 1294-1304.

doi: https://doi.org/10.20546/ijcmas.2019.803.153 